\title{
Resveratrol improves cognition and decreases amyloid plaque formation in Tg6799 mice
}

\author{
YUE CHEN $^{1}$, GUANG-WEI SHI ${ }^{2}$, ZHI-MAN LIANG ${ }^{1}$, SHU-YUE SHENG $^{2}$, YU-SHENG SHI ${ }^{3}$, \\ LI PENG $^{1}$, YA-PING WANG ${ }^{1,4}$, FANG WANG ${ }^{1}$ and XING-MEI ZHANG ${ }^{1}$ \\ ${ }^{1}$ Key Laboratory of Psychiatric Disorders of Guangdong Province, Department of Neurobiology, \\ School of Basic Medical Sciences; ${ }^{2}$ The First Affiliated Hospital; ${ }^{3}$ Department of Radiation Oncology, \\ Nanfang Hospital, Southern Medical University, Guangzhou, Guangdong 510515; ${ }^{4}$ Department of Rehabilitation, \\ Zhujiang Hospital, Southern Medical University, Guangzhou, Guangdong 510282, P.R. China
}

Received May 12,2018; Accepted September 29, 2018

DOI: $10.3892 / \mathrm{mmr} .2019 .10010$

\begin{abstract}
Alzheimer's disease (AD) is an irreversible, progressive neurodegenerative disorder of the central nervous system that causes severe cognitive impairment. One of the most significant pathological features of AD is the accumulation of $\beta$-amyloid (A $\beta$ ) peptide in the brain. Resveratrol (Res) is a polyphenol derived from peanuts, red grapes and other plants, which has received increasing attention due to its neuroprotective features. Tg6799 mice are transgenic mice with five familial AD (FAD) mutations that are also known as 5XFAD mice. The present study aimed to investigate the effects of Res on Tg6799 mice. The transgenic mice were randomly divided into the Res treatment group and the vehicle control group, and were treated with $0.5 \%$ Res solution $(60 \mathrm{mg} / \mathrm{kg})$ or volume-matched normal saline, respectively. Treatment was administered by oral gavage daily for 60 consecutive days. Res reduced amyloid plaque formation and the levels of $A \beta_{42}$, and $\beta$-secretase 1 levels were also significantly decreased. Furthermore, Res was able to reduce the expression of amyloid precursor protein and its cleavage products. The administration of Res to $\operatorname{Tg} 6799$ mice also improved their spatial working memory, as measured by the Y-maze test, and rescued spatial memory deficits, as measured using the Morris water maze test; however, Res did not affect their motor function. In conclusion, this study suggested that Res may reduce $\mathrm{A} \beta$-induced neuronal damage, thus preventing memory loss.
\end{abstract}

Correspondence to: Professor Fang Wang or Professor Xing-Mei Zhang, Key Laboratory of Psychiatric Disorders of Guangdong Province, Department of Neurobiology, School of Basic Medical Sciences, Southern Medical University, 1838 Guangzhou Avenue, Guangzhou, Guangdong 510515, P.R. China

E-mail: fwang@smu.edu.cn

E-mail: zxmray@hotmail.com

Key words: Alzheimer's disease, Tg6799 mice, resveratrol, amyloid plaques, Y-maze test, Morris water maze

\section{Introduction}

Alzheimer's disease (AD) is a progressive neurodegenerative disorder characterized by cognitive and memory impairments (1). There are $>35$ million individuals with $\mathrm{AD}$ worldwide, thus resulting in marked emotional and financial burdens on patients and society (2). The accumulation of $\beta$-amyloid (A $\beta$ ) peptide in the brain is strongly implicated as a main hallmark of sporadic and familial forms of $\operatorname{AD}(3,4)$. Amyloid precursor protein (APP) -derived toxic peptides are found at autopsy in the brains of individuals with $A D(5) . A \beta$ is cleaved from APP by $\beta$-secretase 1 (BACE1) and subsequently by $\gamma$-secretase. BACE1 is considered the rate-limiting enzyme in the production of $A \beta(6,7)$. In addition, accumulation of BACE1 is observed in normal and dystrophic presynaptic terminals surrounding the amyloid plaques in brains of $\mathrm{AD}$ mouse models and patients $(8,9)$.

The effects of medications that are currently approved by the USA Food and Drug Administration for the treatment of AD manifestations are modest, transient and provide only symptomatic treatment (10). Recently, researchers have been paying more attention to herbal extracts for their potential therapeutic effects on AD. Resveratrol (Res) is a natural polyphenol with strong effects, including anti-oxidative, anti-inflammatory, cardiovascular protective, neuroprotective and cancer chemopreventive activities (11-13). It has been suggested that Res can act as a potent antioxidant in neurodegenerative disorders. Previous studies have reported that Res has the ability to regulate $A \beta$ toxicity or significantly increase the $\mathrm{A} \beta$ clearance rate in a mouse model of $\mathrm{AD}(14,15)$. Res can also increase resistance against nerve inflammation and exerts anti-oxidant effects that contribute to its neuroprotective effects on the nervous system $(16,17)$.

$\operatorname{Tg} 6799$ mice are APP/presenelin 1 (PS1) double transgenic mice that coexpress five familial AD (FAD) mutations, which are also known as 5XFAD mice. This mouse strain is a useful model of $\mathrm{AD}$ that recapitulates the relevant pathogenic features of $\mathrm{AD}$ amyloid pathology. Intraneuronal $\mathrm{A} \beta_{42}$ accumulates in the brain starting at 1.5 months, and the levels of brain $A \beta_{42}$ and $A \beta_{40}$ increase with age, whereas, the $A \beta_{42} / A \beta_{40}$ ratio decrease. Amyloid deposition starts at 2 months. Tg6799 mice 
develop memory deficits by 4 months of age, as assessed using a Y-maze test compared with nontransgenic control mice (18). The present study aimed to determine the protective effects and underlying mechanisms of Res in AD treatment.

\section{Materials and methods}

Animals and Res treatment. This study was approved by The Animal Ethics Committee of Nanfang Hospital, Southern Medical University (application no. NFYY-2015-43; Guangzhou, China). The experiments were performed using male Tg6799 mice (Jackson Laboratory, Bar Harbor, ME, USA), which express the human APP and PS1 genes and five FAD mutations on a C57/B6XSJL background (18). These mice were group-housed under standard environmental conditions (12-h light/dark cycle, $23 \pm 1^{\circ} \mathrm{C}$ and $55 \pm 5 \%$ relative humidity), with free access to food and water. The procedures were performed in strict accordance with the National Institutes of Health Guidelines for the Care and Use of Laboratory Animals (19). At 2.5 months of age, 40 male Tg6799 mice (weight, 18-22 g) were randomly divided into the control and Res groups ( $n=20 /$ group). According to a previous study (20), the Res group was treated daily with $0.5 \%$ Res (Sigma-Aldrich; Merck KGaA, Darmstadt, Germany; $60 \mathrm{mg} / \mathrm{kg} /$ day) by oral gavage for 60 consecutive days, whereas the control group was treated with volume-matched normal saline by gavage.

\section{Behavioral testing}

Open field test. The open field apparatus consisted of a rectangular chamber $(40 \times 40 \times 30 \mathrm{~cm})$ made of gray polyvinyl chloride. A video camera, a loudspeaker that provided masking noise and a $25 \mathrm{~W}$ red light bulb (illumination density at the center of the maze was $0.3 \mathrm{~lx}$ ) were placed $180 \mathrm{~cm}$ above the center of the apparatus. The mice were gently placed at the center and were allowed to explore the area for $5 \mathrm{~min}$. The digitized image of the path taken by each mouse was recorded and locomotor activity was subsequently analyzed using EthoVision 7.0 software (Noldus Information Technology, Wageningen, The Netherlands).

$Y$-maze test. A black Y-maze was used with an arm length of $40 \mathrm{~cm}$, width of $3 \mathrm{~cm}$ and wall height of $12.5 \mathrm{~cm}$. Each mouse was placed in the center of the symmetrical Y-maze and allowed to explore freely through the maze until they entered the arms 22 times; the sequence of 22 entries was recorded. Each mouse was placed at the end of one arm facing the center and its movements were recorded for $8 \mathrm{~min}$. Data were analyzed to determine the number of arm entries without repetition. Successful alternations were defined as sequential entries into a new arm. A mouse was excluded from the analysis if no entries into new arms were recorded for 2 consecutive min. Mean alternation percentage $(\%)$ was calculated as the number of successful alternations $/ 20 \times 100$.

Morris water maze. The basic protocol and apparatus for the hidden platform version of the water maze have previously been described (21). Each mouse underwent four trials a day for 6 consecutive days. All mice underwent a probe test for $90 \mathrm{sec}$, where the platform was removed from the pool on day 7. The number of crosses over the platform location (crosses), the time spent in the target quadrant and the speed were recorded using a video tracking system.

Thioflavin S staining. Four mice from each group were sacrificed with $0.75 \%$ pentobarbitone sodium $(50 \mathrm{mg} / \mathrm{kg})$ by intraperitoneal injection. The deeply anesthetized mice exhibiting loss of reflexes and muscle relaxation were perfused with $4 \%$ paraformaldehyde in PBS. Subsequently, the hippocampus was extracted and frozen sections, measuring $35 \mu \mathrm{m}$ in thickness, were prepared at $-25^{\circ} \mathrm{C}$. For thioflavin $\mathrm{S}$ immunostaining, brain sections were allowed to defrost at room temperature and were then washed with PBS for $5 \mathrm{~min}$. The sections were then stained with freshly prepared $0.4 \%$ thioflavin $\mathrm{S}$ solution (Sigma-Aldrich; Merck KGaA) for 5 min in the dark at room temperature, washed twice for 3 min with $70 \%$ alcohol, washed twice for 5 min with PBS and mounted. Image acquisition was performed under a fluorescence microscope. Plaque counts were quantified by counting thioflavin S-positive plaques in the hippocampus. The region of interest was manually selected under $\mathrm{x} 4$ magnification and all parameters were kept constant during the analysis.

$A \beta_{40}$ and $A \beta_{42}$ ELISA. Another four mice from each group were used to conduct A $\beta$ ELISA analyses. Following Res treatment for 60 days, animals were anesthetized by pentobarbitone sodium $(50 \mathrm{mg} / \mathrm{kg})$ and were decapitated. The required amount of homogenization buffer was prepared prior to use. The hemisphere tissues $(50 \mathrm{mg})$ were isolated and homogenized using a Potter-Elvehjem homogenizer (DWK Life Sciences, Millville, NJ, USA). Brain homogenates were treated with PBS/protease inhibitor (Thermo Fisher Scientific, Inc., Waltham, MA, USA) and were supplemented with guanidine $\mathrm{HCl}$ to a final concentration of $5 \mathrm{~mol} / \mathrm{l}$. The brain tissue samples were then added to an Eppendorf tube containing the brain extraction buffer provided in the ELISA kits (cat. nos. KHB3481, KHB3441 and KHB3442; Invitrogen; Thermo Fisher Scientific, Inc.). The sample was ground thoroughly with a tissue homogenizer, and then centrifuged at $16,000 \mathrm{xg}$ for $20 \mathrm{~min}$ at $4^{\circ} \mathrm{C}$. The supernatant was carefully transferred to a new tube and diluted with the buffer provided in the BioRad protein assay kit (Bio-Rad Laboratories, Inc., Hercules, CA, USA). Final guanidine $\mathrm{HCl}$ concentrations were $<0.1 \mathrm{~mol} / \mathrm{l}$. The protein content was measured using the Bio-Rad protein assay kit. Duplicate samples were subsequently analyzed using $\mathrm{A} \beta_{40^{-}}$and $\mathrm{A} \beta_{42}$-specific sandwich colorimetric ELISA kits (cat. nos. KHB3481, KHB3441 and KHB3442; Invitrogen; Thermo Fisher Scientific, Inc.), according to the manufacturer's protocol. The absorbance was measured at $450 \mathrm{~nm}$, and curve-fitting software (TableCurve 2D; version 6; Jandel Scientific Software, San Rafael, CA) was used to generate the standard curve.

Western blotting. For western blot analysis, hippocampal tissues were dissected from four mice in each group and disrupted by sonication (three pulses at $1 \mathrm{~Hz}$ for $20 \mathrm{sec}$ with $30 \mathrm{sec}$ intervals between pulses) for $2 \mathrm{~min}$ in ice-cold radioimmunoprecipitation assay buffer with a complete protease inhibitor cocktail (Roche Diagnostics, Indianapolis, IN, USA). The lysates were centrifuged at $16,900 \mathrm{x}$ g for $40 \mathrm{~min}$ at $4^{\circ} \mathrm{C}$, and the supernatant was boiled in loading buffer for $10 \mathrm{~min}$. 

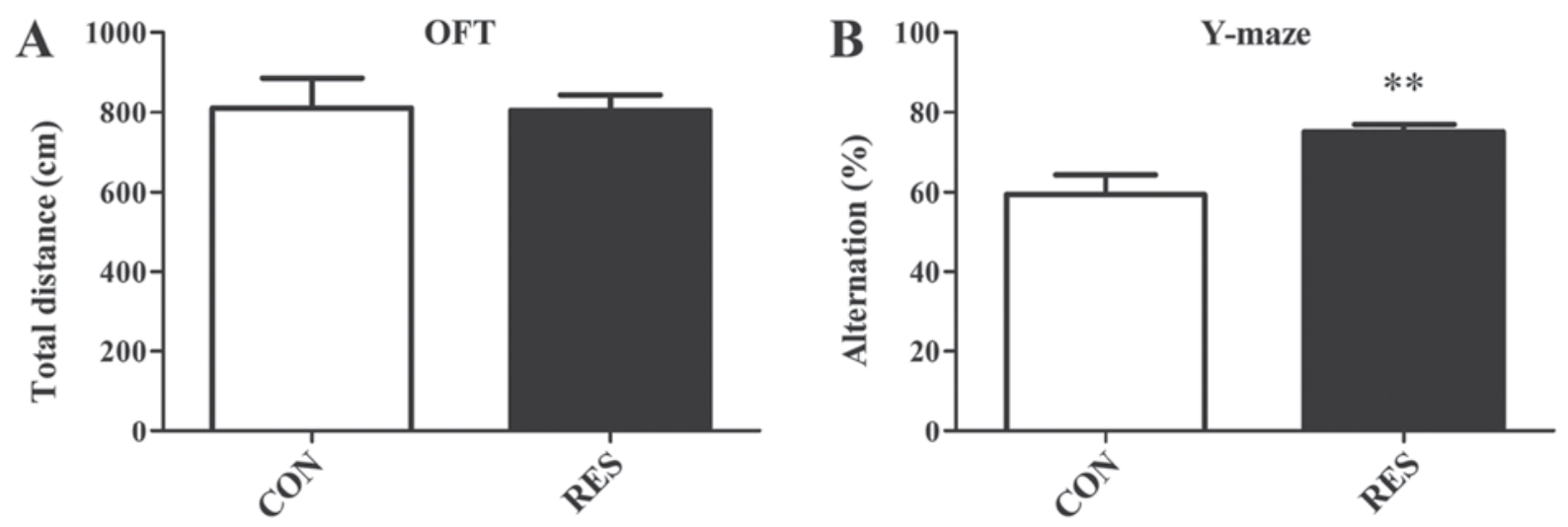

Figure 1. Res treatment improves spatial working memory in the Tg6799 mouse model of Alzheimer's disease. (A) Total distance of the mice in the control and Res groups in the OFT. (B) Spontaneous alternation in the Y-maze test. Data are presented as the means \pm standard error of the mean. ${ }^{* *} \mathrm{P}<0.01$ vs. the control group. OFT, open field test; Res, resveratrol.

The protein content was measured using a Bio-Rad protein assay kit (Bio-Rad Laboratories, Inc.). A total of $50 \mu \mathrm{g}$ protein was loaded into each lane and separated by SDS-PAGE with $10 \%$ polyacrylamide gels. Following transfer onto Immobilon polyvinylidene difluoride membranes, nonspecific binding was blocked with $5 \%$ nonfat milk in TBS containing $0.05 \%$ Tween 20 for $1.5 \mathrm{~h}$ at room temperature. After the membranes were washed, they were incubated with the following primary antibodies: Anti-APP (1:1,000; cat. no. SIG-39300; BioLegend, Inc., San Diego, CA, USA), anti-BACE1 (1:1,000; cat. no. ab108394; Abcam, Cambridge, UK), anti-secreted (s)APP $\alpha$ (1:500; cat. no. 2B3; IBL, Japan), anti-sAPP $\beta$ (1:500, cat. no. 6A1; Immuno-Biological Laboratories Co., Ltd., Fujioka, Japan) and anti-sirtuin 1 (SIRT1; 1:1,000; cat. no. 8469s; Cell Signaling Technology, Inc., Danvers, MA, USA) at $4^{\circ} \mathrm{C}$ overnight. The membranes were then incubated with horseradish peroxidase-conjugated goat anti-mouse or anti-rabbit secondary antibodies (1:2,000; cat. nos. TA130003 and TA130023; OriGene Technologies, Inc., Beijing, China) for $1 \mathrm{~h}$ at room temperature. Bands were visualized using enhanced chemiluminescence western blot detection reagents (Bio-Rad Laboratories, Inc.). ImageJ software (version 1.50; National Institutes of Health, Bethesda, MD, USA) was used to semi-quantify the relative expression levels of target proteins, which were normalized to the internal control GAPDH (1:1,000; cat. no. 5174; Cell Signaling Technology, Inc.).

Statistical analysis. Data are presented as the means \pm standard error of the mean, and all experiments were repeated at least three times. Data analysis was conducted using SPSS 18.0 statistical software (SPSS, Inc., Chicago, IL, USA). Comparisons between two groups were performed using two independent samples Student's t-test. $\mathrm{P}<0.05$ was considered to indicate a statistically significant difference.

\section{Results}

Res treatment rescues cognitive impairment but does not affect the motor function of Tg6799 mice. Open field tests were used to measure locomotor activity, which served as a control to reflect motor function. In the open field test, the total distances of the two groups over $5 \mathrm{~min}$ were not significantly different (Fig. 1A). In addition, the spontaneous alternation performance of mice was determined using the Y-maze test, which is used to assess their spatial working memory. The results revealed that the Res group had an elevated mean alternation percentage compared with the control group (Fig. 1B). These findings indicated that the administration of Res improved spatial working memory.

The effects of Res on learning and memory were also determined in AD mice using the Morris water maze. The Morris water maze test is most widely used to test spatial memory in AD mouse models. Over the 6-day training phase, the escape latencies of all mice gradually decreased; however, the Res group mice exhibited significantly lower escape latencies compared with the controls (Fig. 2A). The average swimming speed during training and the probe test did not differ between the two groups (Fig. 2B). In addition, the administration of Res significantly increased the number of crosses and time spent in the target quadrant (Fig. 2C and D). The swimming path during the training phase and the probe test reflected the improved learning and memory of the mice in the Res group (Fig. 2E and F).

Administration of Res reduces amyloid plaques and $A \beta_{42}$ levels in Tg6799 mice. Thioflavin S staining was performed to examine amyloid plaque pathology. Amyloid deposition was positive after staining with thioflavin $\mathrm{S}$. The results revealed that the plaque loads in the Res group were quantitatively lower compared with in the control group. Amyloid plaques were significantly reduced in the hippocampus of the Res group compared with in the control group (Fig. 3A and B). Cerebral levels of $A \beta_{42}$ and $A \beta_{40}$ were also detected in hippocampal homogenates using ELISA kits. The Res group had lower levels of $A \beta_{42}$ and $A \beta_{40}$ compared with the control group. For $A \beta_{42}$ levels, this alteration was statistically significant (Fig. 3C and D), which was consistent with the thioflavin $\mathrm{S}$ staining results.

Administration of Res alters the levels of BACE1, APP, $s A P P \alpha$ and $s A P P \beta$ in Tg6799 mice. Western blotting was used to determine APP, cleaved APP products and BACE1 expression, in order to investigate the mechanism underlying the reduction of amyloid plaques in the Res group. The results of western 

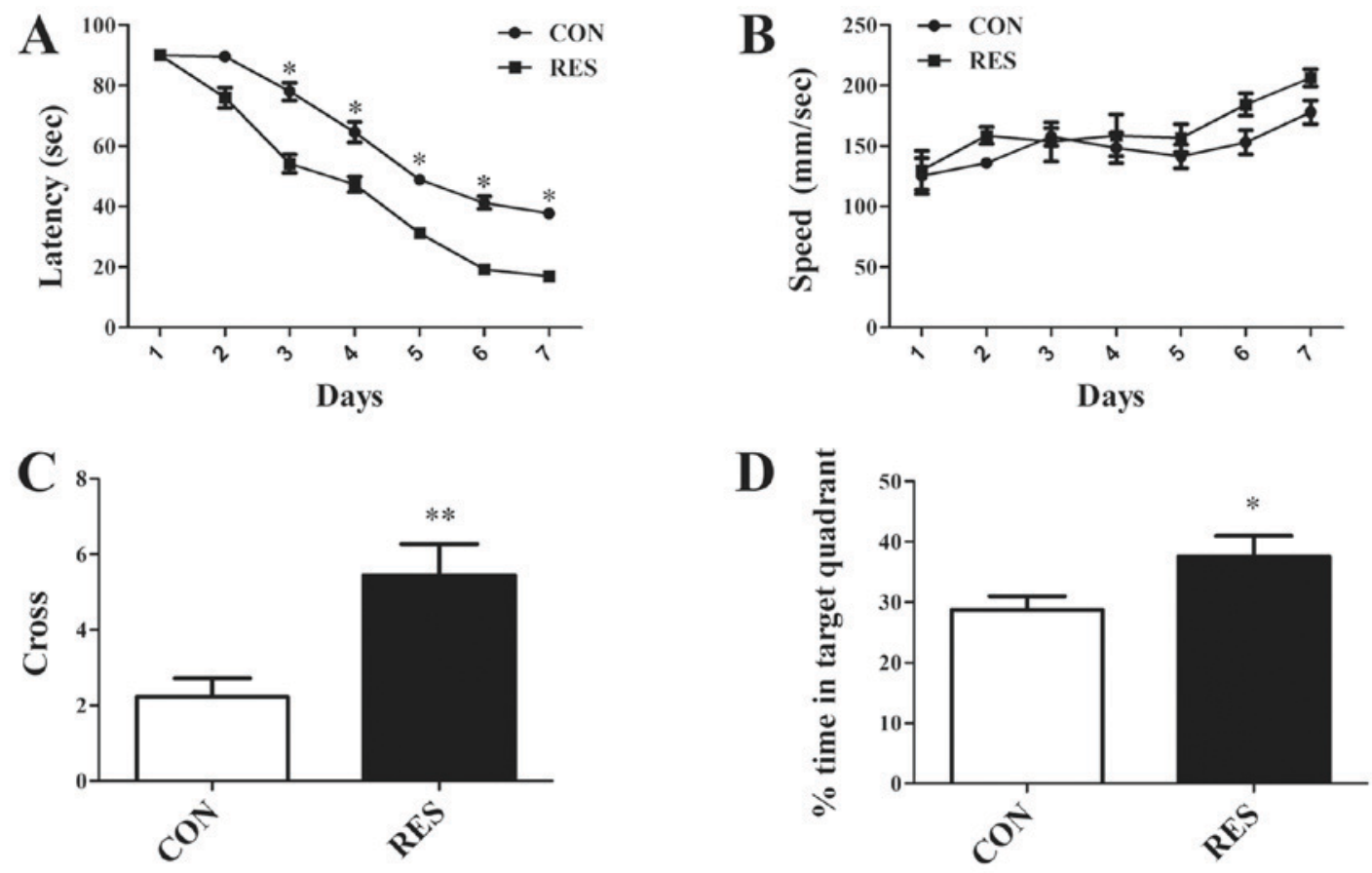

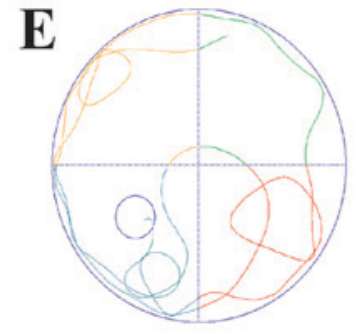

CON

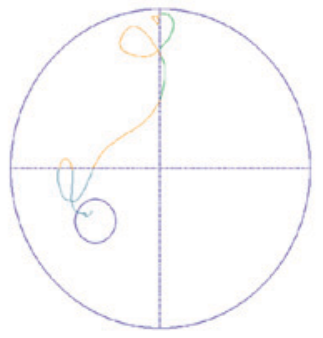

RES

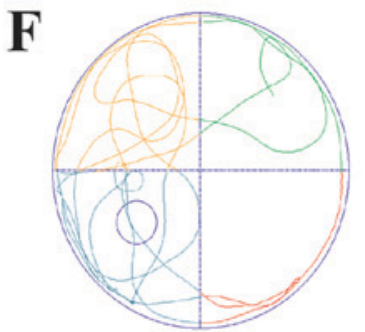

CON

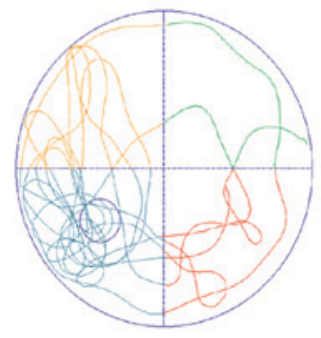

RES

Figure 2. Res treatment rescues impaired water maze learning in the Tg6799 mouse model of Alzheimer's disease. (A) Escape latency during the training phase and the probe test. (B) Average swimming speed. (C) Number of crosses in the probe test. (D) Percentage of time spent in the target quadrant in the probe test. (E) A representative swimming path on day 6 of training. (F) A representative swimming path during the probe test. Data are presented as the means \pm standard error of the mean. ${ }^{*} \mathrm{P}<0.05,{ }^{* *} \mathrm{P}<0.01$ vs. the control group. Res, resveratrol.

blot analysis revealed that the expression levels of APP, sAPP $\alpha$, sAPP $\beta$ and BACE1 were significantly reduced in the Res group compared with in the control group (Fig. 4).

Res treatment did not affect SIRT1 levels in Tg6799 mice. A previous study (22) suggested that activation of the sirtuin family member SIRT1 is an important pharmacological effect of Res. Therefore, SIRT1 levels were determined by western blotting; however, the administration of Res did not affect the protein expression levels of SIRT1 (Fig. 5).

\section{Discussion}

The pathogenesis of AD continues to be debated, and there are numerous hypotheses, including the amyloid cascade hypothesis, neurotrophic factor hypothesis and oxidative stress hypothesis (23). The pathological hallmarks of AD include intraneuronal neurofibrillary tangles, extracellular amyloid plaques, neuronal loss and gliosis. The aggregation of $\mathrm{A} \beta$ forms amyloid plaques. Numerous clinical, cytobiological and experimental animal studies $(24,25)$ support the causative role of $A \beta$ in the pathogenesis of AD. Notably, $A \beta$ can induce loss of synaptic terminals and calcium imbalance, and can drive tau aggregation, thus inducing neurofibrillary tangles $(26,27)$. $\mathrm{A} \beta$ is cleaved from APP, genetic mutations of which cause a familial form of AD. BACE1 cuts APP at the N-terminus of the $\mathrm{A} \beta$ domain to produce $\mathrm{C} 99$ and sAPP $\beta$, after which, $\mathrm{C} 99$ is further cleaved by $\gamma$-secretase to generate $\mathrm{A} \beta(28)$. Notably, previous studies have demonstrated that Res is able to cross the blood-brain barrier, thus making oral gavage an efficient route of administration $(29,30)$.

The present study demonstrated that the administration of Res significantly diminished the number and intensity of amyloid plaques, as measured by thioflavin S staining. Senile plaques are formed by the accumulation of APP-derived toxic peptides (predominantly $A \beta_{42}$ ). Previous data support the hypothesis that $\mathrm{AD}$ neurodegeneration is initiated by an imbalance between $A \beta_{42}$ production and clearance in selected brain regions (4). $\operatorname{Tg} 6799$ mice accumulate large amounts of cerebral $A \beta_{42}$ at a young age, and generate much more $A \beta_{42}$ than $A \beta_{40}$. The ELISA results in this study demonstrated that the cerebral levels of $A \beta_{42}$ and $A \beta_{40}$ were decreased in hippocampal homogenates in response to Res, particularly $A \beta_{42}$ levels, which were significantly reduced. 
A
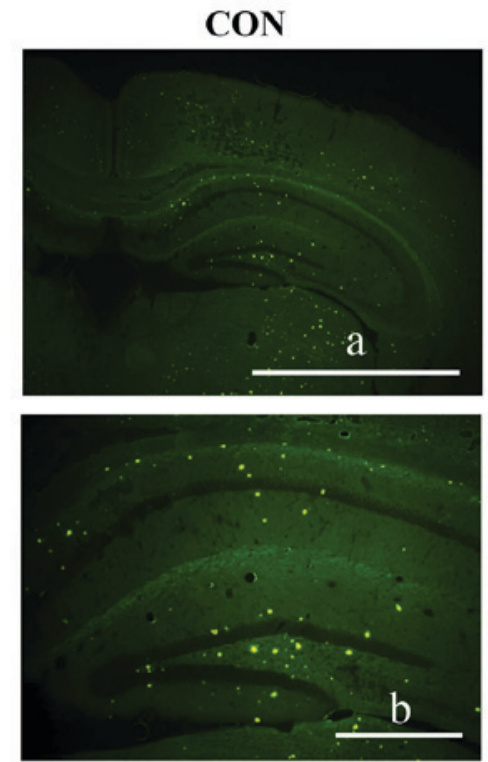

RES
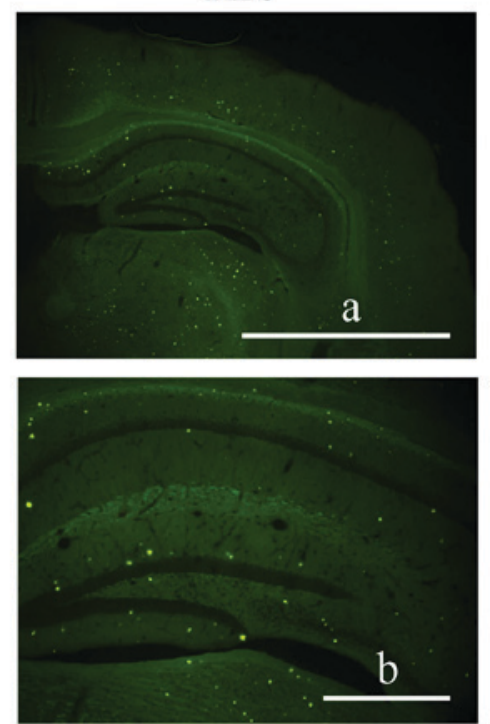

B

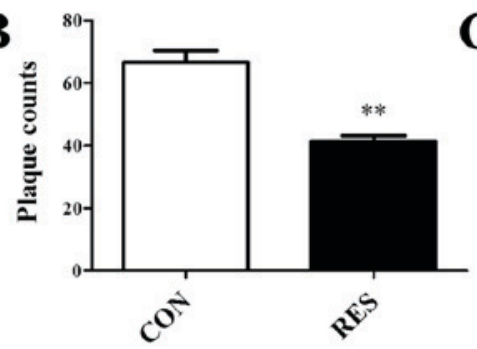

C

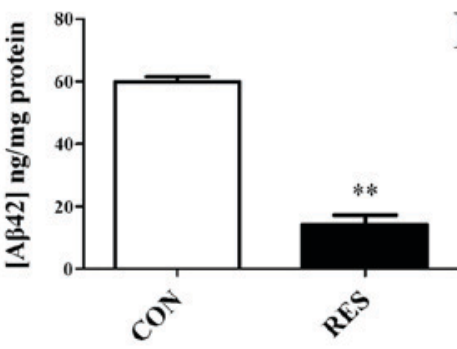

D

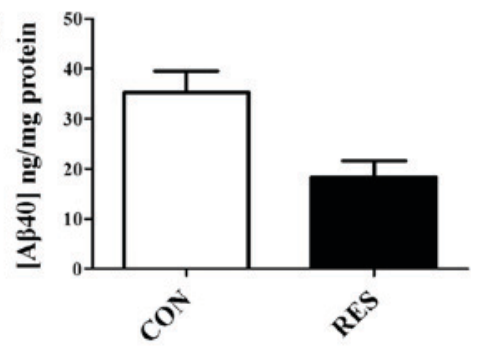

Figure 3. Res treatment reduces amyloid plaques in the Tg6799 mouse model of Alzheimer's disease. (A) Representative images of thioflavin S staining of amyloid plaques in mouse brains. Scale bar: $A=2,000 \mu \mathrm{m}, \mathrm{b}=500 \mu \mathrm{m}$. (B) Quantification of the number of amyloid plaques in the hippocampus. (C and D) Cerebral levels of $\mathrm{A} \beta_{42}$ and $\mathrm{A} \beta_{40}$ in hippocampal homogenates. Data are presented as the means \pm standard error of the mean. ${ }^{* *} \mathrm{P}<0.01$ vs. the control group. $\mathrm{A} \beta$, $\beta$-amyloid; Res, resveratrol.

In vitro, Res promotes $\mathrm{A} \beta$ clearance potentially by increasing intracellular proteasomal activity (31). In a previous study, the $\mathrm{Tg} 2576$ mouse transgenic model of $\mathrm{AD}$ was fed red wine (containing Res), after which cognitive improvement or attenuation of amyloid brain pathology was assessed based on nonamyloidogenic processing of $\beta$ APP or the oligomerization of $A \beta$ molecules $(15,32)$. To investigate the mechanism underlying the reduction of plaque pathology by Res, the present study detected the expression levels of APP-associated enzymes by western blotting. A significant decrease in BACE1 levels may alter the APP processing pathway, leading to a reduction in amyloid burden. BACE1 is considered the rate-limiting enzyme in the production of A $\beta$. High-molecular weight APP and sAPP $\alpha$ levels were also decreased in response to Res. A number of previous studies $(33,34)$ have suggested that Res can promote $A \beta$ clearance without affecting $A \beta$-producing enzyme activities $(\gamma$-secretase and BACE1). This mechanism could be synergistic with a reduction in BACE1.

A previous study (35) suggested that Res is able to induce activation of the sirtuin family member SIRT1, which is beneficial for preventing neurodegeneration and improving memory. In addition, Res can counteract $A \beta$ toxicity in cellular models due to its natural antioxidant properties and SIRT1 activation (36-38). Other findings have suggested that Res is able to reduce the harmful process that occurs in the
APP/PS1 mouse hippocampus, which is mainly mediated by increased activation of SIRT1 and 5' AMP-activated protein kinase pathways (39). However, in the present study, Res did not induce activation of SIRT1.

The present study assessed hippocampus-dependent spatial working memory in mice using the Y-maze test. This learning task does not involve any training, reward or punishment, and allows for the assessment of hippocampus-dependent spatial working memory. The hippocampus is the hub for learning and memory. The results demonstrated that treatment with Res resulted in a significant increase in the mean alternation percentage. It may be hypothesized that reduced amyloid plaques are responsible for the improved working memory in the Res group. Furthermore, the effects of Res treatment on learning and memory in $\operatorname{Tg} 6799$ mice were evaluated using the Morris water maze test. In the hidden platform version of the water maze test, animals learn to locate a submerged platform in a pool filled with opaque water. This spatial navigation performance is also known to be hippocampus dependent. This study confirmed that learning and memory were significantly improved by the administration of Res.

Numerous previous studies $(40,41)$ have described the neuroprotective properties of Res; however, Res treatment as a therapy for AD still has many limitations, including poor bioavailability. The complex interaction and mechanisms 

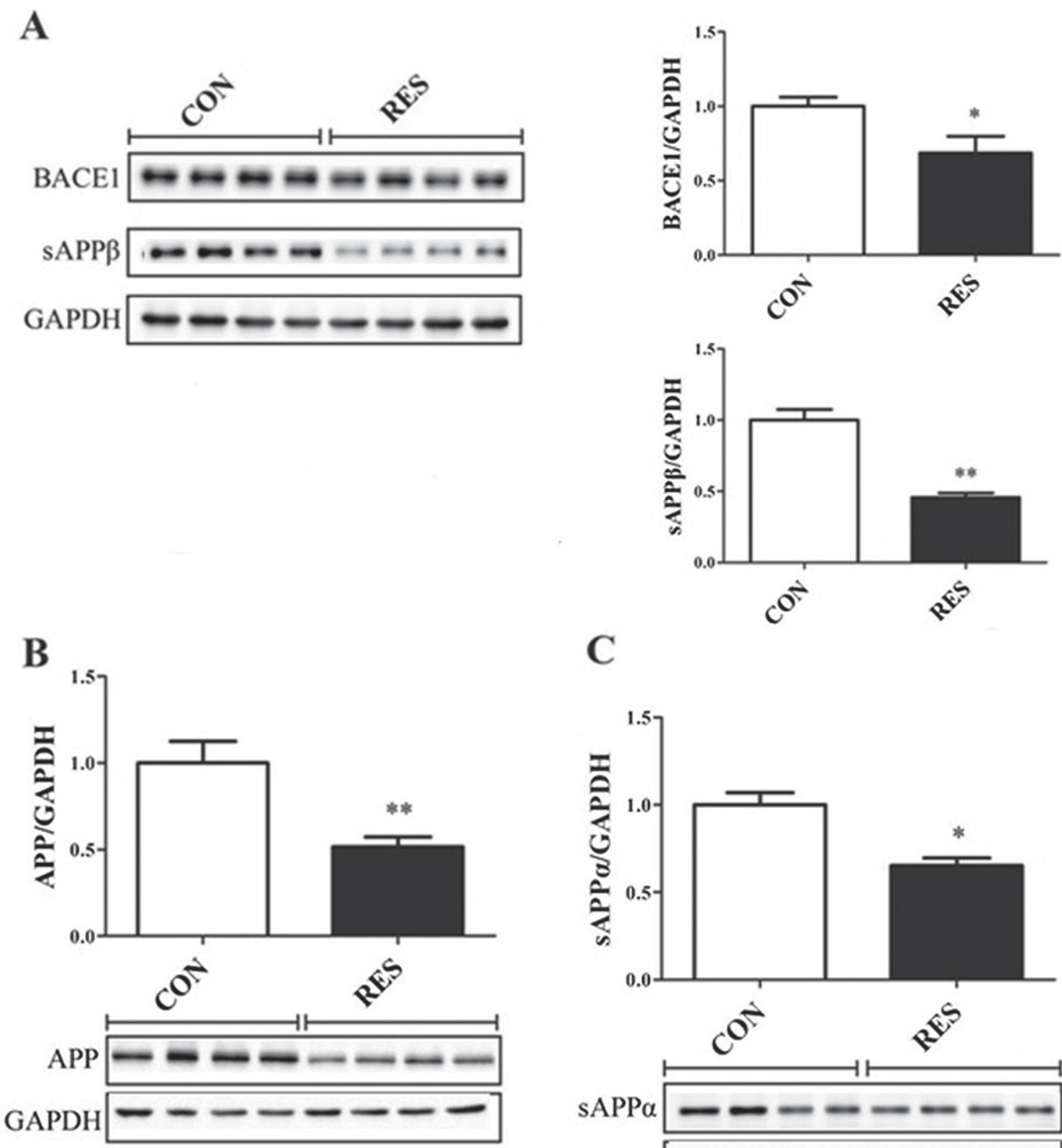

C

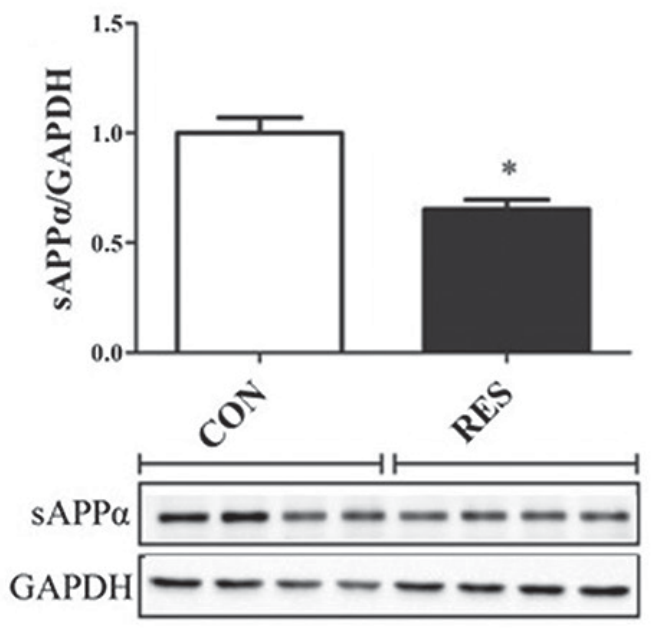

Figure 4. Western blot analysis of (A) BACE1 and sAPP $\beta$, (B) APP and (C) SAPP $\alpha$. Data are presented as the means \pm standard error of the mean. "P<0.05, ${ }^{* *} \mathrm{P}<0.01$ vs. the control group. APP, amyloid precursor protein; BACE1, $\beta$-secretase 1 ; Res, resveratrol; sAPP, secreted APP.

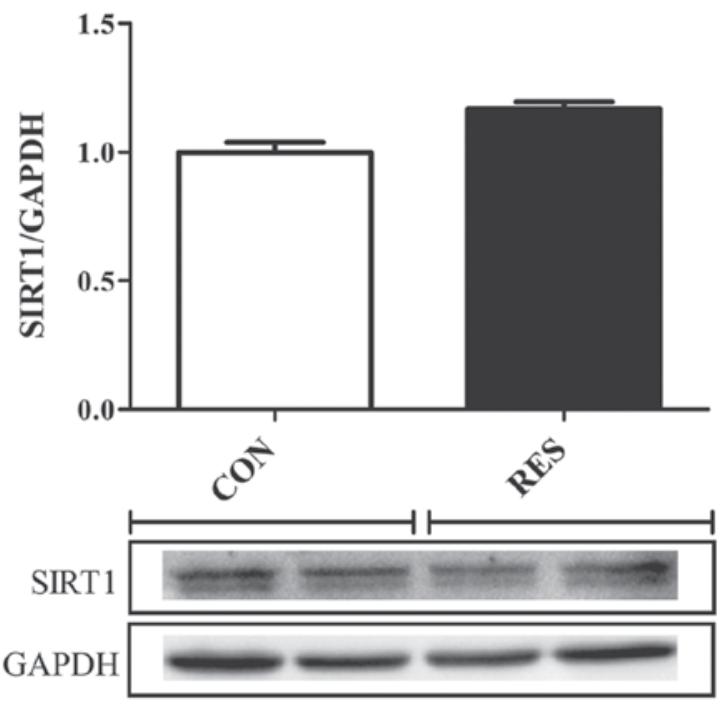

Figure 5. Western blot analysis of SIRT1 levels. Data are presented as the means \pm standard error of the mean. Res, resveratrol; SIRT1, sirtuin 1 . of Res remain to be explored. In future studies, we aim to investigate the expression of $\alpha$ and $\gamma$ secretases (ADAM metallopeptidase domain 9, 10 and 17, and PS1), and to investigate whether a modified treatment with Res may induce alterations in SIRT1 activity. In the present study, mice were treated with $60 \mathrm{mg} / \mathrm{kg} /$ day Res by oral gavage; this dosage per day is much higher than that ingested by humans via diet and wine, this is also an important issue to be considered in future work.

In conclusion, the present study demonstrated that the administration of Res for 60 consecutive days protected against A $\beta$ plaque formation and cognitive loss in Tg6799 mice. The exact underlying mechanism has yet to be elucidated; however, the animal behavioral data suggested that Res may serve a neuroprotective role in a mouse model of AD.

\section{Acknowledgements}

Not applicable. 


\section{Funding}

The present study was supported by the National Natural Science Foundation of China (grant nos. 81471388 and 81771484), the Natural Science Foundation of Guangdong Province, China (grant nos. 2015A030313235 and 2014A030313351), the Guangdong Science \& Technology Planning Project, China (grant no. 2014A020211016), the Undergraduate Innovation and Entrepreneurship Training Program of Southern Medical University (grant no. C1051222), and the Program for Changjiang Scholars and Innovative Research Team in University (grant no. IRT_16R37).

\section{Availability of data and materials}

All data generated or analyzed during the present study are included in this published article.

\section{Authors' contributions}

$\mathrm{XMZ}, \mathrm{FW}$ and YC conceived and designed the experiments, and revised the final version of the paper. YC, ZML and GWS performed the experiments. YC and YSS analyzed the data and wrote the paper. LP, SYS and YPW prepared reagents and materials, and performed part of the experiments. All authors read and approved the final manuscript.

\section{Ethics approval and consent to participate}

This study was approved by the animal ethics committee of Nanfang Hospital, Southern Medical University (application no. NFYY-2015-43).

\section{Patient consent for publication}

Not applicable.

\section{Competing interests}

The authors declare that they have no competing interests.

\section{References}

1. McKhann G, Drachman D, Folstein M, Katzman R, Price D and Stadlan EM: Clinical diagnosis of Alzheimer's disease: Report of the NINCDS-ADRDA work group under the auspices of department of health and human services task force on Alzheimer's disease. Neurology 34: 939-944, 1984.

2. Alzheimer's Association: 2011 Alzheimer's disease facts and figures. Alzheimers Dement 7: 208-244, 2011.

3. Selkoe DJ and Schenk D: Schenk, Alzheimer's disease: Molecular understanding predicts amyloid-based therapeutics. Annu Rev Pharmacol Toxicol 43: 545-584, 2003.

4. Hardy J and Selkoe DJ: The amyloid hypothesis of Alzheimer's disease: Progress and problems on the road to therapeutics. Science 297: 353-356, 2002.

5. Grundke-Iqbal I, Iqbal K, Tung YC, Quinlan M, Wisniewski HM and Binder LI: Abnormal phosphorylation of the microtubule-associated protein tau (tau) in Alzheimer cytoskeletal pathology. Proc Natl Acad Sci USA 83: 4913-4917, 1986.

6. Cole SL and Vassar R: BACE1 structure and function in health and Alzheimer's disease. Curr Alzheimer Res 5: 100-120, 2008.

7. Lin X, Koelsch G, Wu S, Downs D, Dashti A and Tang J: Human aspartic protease memapsin 2 cleaves the beta-secretase site of beta-amyloid precursor protein. Proc Natl Acad Sci USA 97: 1456-1460, 2000.
8. Zhao J, Fu Y, Yasvoina M, Shao P, Hitt B, O'Connor T, Logan S, Maus E, Citron M, Berry R, et al: Beta-site amyloid precursor protein cleaving enzyme 1 levels become elevated in neurons around amyloid plaques: Implications for Alzheimer's disease pathogenesis. J Neurosci 27: 3639-3649, 2007.

9. Kandalepas PC, Sadleir KR, Eimer WA, Zhao J, Nicholson DA and Vassar R: The Alzheimer's $\beta$-secretase BACE1 localizes to normal presynaptic terminals and to dystrophic presynaptic terminals surrounding amyloid plaques. Acta Neuropathol 126: 329-352, 2013.

10. De Strooper B: Proteases and proteolysis in Alzheimer disease: A multifactorial view on the disease process. Physiol Rev 90: 465-494, 2010

11. Robb EL, Page MM, Wiens BE and Stuart JA: Molecular mechanisms of oxidative stress resistance induced by resveratrol: Specific and progressive induction of MnSOD. Biochem Biophys Res Commun 367: 406-412, 2008.

12. Baltaci AK, Arslangil D, Mogulkoc R and Patlar S: Effect of resveratrol administration on the element metabolism in the blood and brain tissues of rats subjected to acute swimming exercise. Biol Trace Elem Res 175: 421-427, 2017.

13. Baltaci SB, Mogulkoc R and Baltaci AK: Resveratrol and Exercise. Biomed Rep 5: 525-530, 2016.

14. Ladiwala AR, Lin JC, Bale SS, Marcelino-Cruz AM, Bhattacharya M, Dordick JS and Tessier PM: Resveratrol selectively remodels soluble oligomers and fibrils of amyloid Abeta into off-pathway conformers. J Biol Chem 285: 24228-24237, 2010.

15. Wang J, Ho L, Zhao Z, Seror I, Humala N, Dickstein DL, Thiyagarajan M, Percival SS, Talcott ST and Pasinetti GM: Moderate consumption of Cabernet Sauvignon attenuates Abeta neuropathology in a mouse model of Alzheimer's disease. FASEB J 20: 2313-2320, 2006.

16. Capiralla H, Vingtdeux V, Zhao H, Sankowski R, Al-Abed Y, Davies P and Marambaud P: Resveratrol mitigates lipopolysaccharide- and $\mathrm{A} \beta$-mediated microglial inflammation by inhibiting the TLR4/NF- $\mathrm{KB} / \mathrm{STAT}$ signaling cascade. J Neurochem 120: 461-472, 2012.

17. Crandall JP and Barzilai N: Exploring the promise of resveratrol: Where do we go from here? Diabetes 62: 1022-1023, 2013.

18. Oakley H, Cole SL, Logan S, Maus E, Shao P, Craft J, Guillozet-Bongaarts A, Ohno M, Disterhoft J, Van Eldik L, et al: Intraneuronal beta-amyloid aggregates, neurodegeneration, and neuron loss in transgenic mice with five familial Alzheimer's disease mutations: Potential factors in amyloid plaque formation. J Neurosci 26: 10129-10140, 2006.

19. The NIH guidelines for the care and use of laboratory animals NIH Publication 85-23, revised 1985, 1985.

20. Menet MC, Baron S, Taghi M, Diestra R, Dargère D, Laprévote O, Nivet-Antoine V, Beaudeux JL, Bédarida T and Cottart CH: Distribution of trans-resveratrol and its metabolites after acute or sustained administration in mouse heart, brain, and liver. Mol Nutr Food Res 61, 2017.

21. Ohno M, Tseng W, Silva AJ and Disterhoft JF: Trace eyeblink conditioning requires the hippocampus but not autophosphorylation of alphaCaMKII in mice. Learn Mem 12: 211-215, 2005.

22. Hong YA, Bae SY, Ahn SY, Kim J, Kwon YJ, Jung WY and Ko GJ: Resveratrol ameliorates contrast induced nephropathy through the activation of SIRT1-PGC- $1 \alpha$-Foxo1 signaling in mice. Kidney Blood Press Res 42: 641-653, 2017.

23. Area-Gomez E and Schon EA: Alzheimer disease. Adv Exp Med Biol 997: 149-156, 2017.

24. Barage SH and Sonawane KD: Amyloid cascade hypothesis: Pathogenesis andtherapeutic strategies in Alzheimer's disease. Neuropeptides 52: 1-18, 2015.

25. Andreeva TV, Lukiw WJ and Rogaev EI: Biological basis for amyloidogenesis in Alzheimer's disease. Biochemistry (Mosc) 82: 122-139, 2017.

26. Billings LM, Oddo S, Green KN, McGaugh JL and LaFerla FM: Intraneuronal Abeta causes the onset of early Alzheimer's disease-related cognitive deficits in transgenic mice. Neuron 45: 675-688, 2005.

27. Selkoe DJ and Schenk D: Alzheimer's disease: Molecular understanding predicts amyloid-based therapeutics. Annu Rev Pharmacol Toxicol 43: 545-584, 2003.

28. Fukumoto H, Takahashi H, Tarui N, Matsui J, Tomita T, Hirode M, Sagayama M, Maeda R, Kawamoto M, Hirai K, et al: A noncompetitive BACE1 inhibitor TAK-070 ameliorates Abeta pathology and behavioral deficits in a mouse model of Alzheimer's disease. J Neurosci 30: 11157-11166, 2010.

29. Han YS, Bastianetto S, Dumont Y and Quirion R: Specific plasma membrane binding sites for polyphenols, including resveratrol, in the rat brain. J Pharmacol Exp Ther 318: 238-245, 2006. 
30. Gilgun-Sherki Y, Melamed E and Offen D: Oxidative stress induced-neurodegenerative diseases: The need for antioxidants that penetrate the blood brain barrier. Neuropharmcology 40: 959-975, 2001.

31. Marambaud P, Zhao H and Davies P: Resveratrol promotes clearance of Alzheimer's disease amyloid-beta peptides. J Biol Chem 280: 37377-37382, 2005.

32. Ho L, Chen LH, Wang J, Zhao W, Talcott ST, Ono K, Teplow D, Humala N, Cheng A, Percival SS, et al: Heterogeneity in red wine polyphenolic contents differentially influences Alzheimer's disease-type neuropathology and cognitive deterioration. J Alzheimers Dis 16: 59-72, 2009.

33. Jia Y, Wang N and Liu X: Resveratrol and amyloid-beta: Mechanistic insights. Nutrients 9: pii: E1122, 2017.

34. Deng $\mathrm{H}$ and Mi MT: Resveratrol attenuates A $\beta 25-35$ caused neurotoxicity by inducing autophagy through the TyrRS-PARP1-SIRT1 signaling pathway. Neurochem Res 41: 2367-2379, 2016.

35. Chen CJ, Yu W, Fu YC, Wang X, Li JL and Wang W: Resveratrol protects cardiomyocytes from hypoxia-induced apoptosis through the SIRT1-FoxO1 pathway. Biochem Biophys Res Commun 378: 389-393, 2009.
36. Jang JH and Surh YJ: Protective effect of resveratrol on beta-amyloid-induced oxidative PC12 cell death. Free RadicBiol Med 34: 1100-1110, 2003

37. Kim D, Nguyen MD, Dobbin MM, Fischer A, Sananbenesi F, Rodgers JT, Delalle I, Baur JA, Sui G, Armour SM, et al: SIRT1 deacetylase protects against neurodegeneration in models for Alzheimer's disease and amyotrophic lateral sclerosis. EMBO J 26: 3169-3179, 2007

38. Lagouge M, Argmann C, Gerhart-Hines Z, Meziane H, Lerin C, Daussin F, Messadeq N, Milne J, Lambert P, Elliott P, et al: Resveratrol improves mitochondrial function and protects against metabolic disease by activating SIRT1 and PGC-1alpha. Cell 127: 1109-1122, 2006.

39. Porquet D, Griñán-Ferré C, Ferrer I, Camins A, Sanfeliu C, Del Valle J and Pallàs M: Neuroprotective role of trans-resveratrol in a murine model of familial Alzheimer's disease. J Alzheimers Dis 42: 1209-1220, 2014.

40. Bastianetto S, Ménard C and Quirion R: Neuroprotective action of resveratrol. Biochim Biophys Acta 1852: 1195-1201, 2015.

41. Hou Y,Wang K, Wan W, Cheng Y,PuXand YeX:Resveratrolprovides neuroprotection by regulating the JAK2/STAT3/PI3K/AKT/mTOR pathway after stroke in rats. Genes Dis 5: 245-255, 2018. 